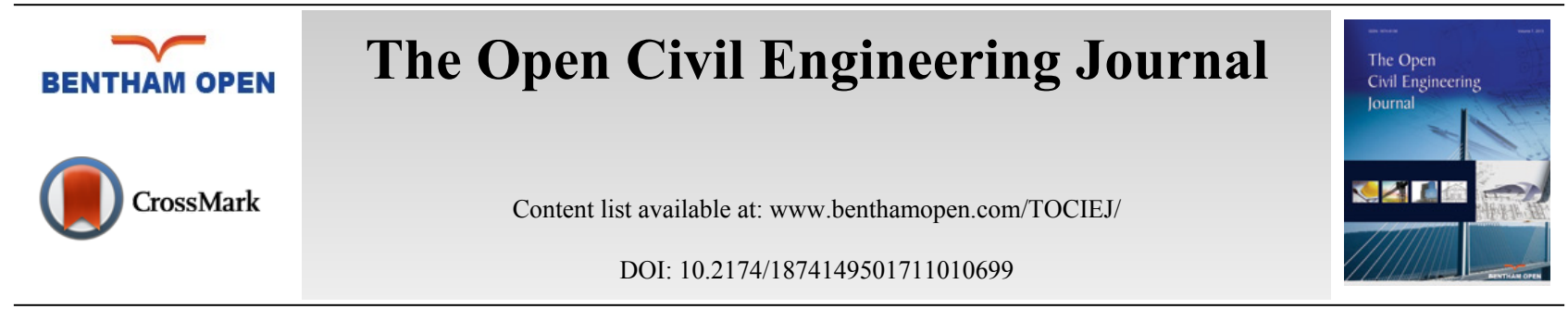

RESEARCH ARTICLE

\title{
Dynamic Response of RC Frame Structures Under the Action of Mining Blasting
}

\author{
Xuansheng Cheng ${ }^{1,2, *}$, Yingchao Zhou, ${ }^{2}$ Chao $\mathrm{Bao}^{2}$ and Jiexuan $\mathrm{Ma}^{1,2}$ \\ ${ }^{I}$ Key Laboratory of Disaster Prevention and Mitigation in Civil Engineering of Gansu Province, Lanzhou University of \\ Technology, Lanzhou, 730050, PR China \\ ${ }^{2}$ Western Engineering Research Center of Disaster Mitigation in Civil Engineering of Ministry of Education, Lanzhou \\ University of Technology, Lanzhou, 730050, PR China
}

Received: February 27, 2017

Revised: June 02, 2017

Accepted: June 15, 2017

\begin{abstract}
:
Introduction:

In this study, the acceleration time-history curve of blasting vibration is obtained at different explosion distances in different amount of explosion with MATLAB analysis software to determine the dynamic response of mining blasting vibration to surrounding reinforced concrete $(\mathrm{RC})$ frame structures.
\end{abstract}

\section{Objective:}

Moreover, this paper discusses the dynamic response of two RC structures with different numbers of stories that happen to blasting vibration waves generated by using different amount of explosion at different blasting distances.

\section{Result:}

Results show that the vibration response of the RC frame structures may be larger under the action of blasting vibration. The smaller the explosion source distance, the lower is the structure, and the larger is the story drift, that is, in general, with the same explosion charge and small explosion source, the deformation of lower structure is larger than the higher. The larger the explosion source distance, the smaller is the change range of floor displacement. A larger distance results in a weaker blasting vibration response.

\section{Conclusion:}

Furthermore, the acceleration amplitudes of the top stories are almost equal in both structures with the same amount of explosion and the same explosion distance. It is suggested that the higher the structure, the greater is the influence of explosion source distance on the change rate of floor displacement and story drift. Therefore, allowable safety distance should be determined according to the mining conditions and structural height during structure design.

Keywords: Mining, Blasting, Reinforced concrete, Frame structure, Dynamic response.

\section{INTRODUCTION}

Blasting technology plays an increasingly important role in the process of mining. Blasting vibration and damage on adjacent buildings are common, and damage to buildings by mining blasting is affected by several factors. These factors include the characteristics of the blasting wave, the composition of the structure, construction quality, and the characteristics of foundation soil on site. The generation of a blasting wave involves a process of random vibration. Therefore, the destruction of accurately estimated buildings by blasting waves is a complicated problem. Guaranteeing

\footnotetext{
* Address correspondence to this author at Key Laboratory of Disaster Prevention and Mitigation in Civil Engineering of Gansu Province, Lanzhou University of Technology, Lanzhou, 730050, PR China; Tel: +86-931-2973784; E-mail: cxs702@126.com
} 
the safety of buildings around a mining area is thus an urgent problem.

Existing studies have examined the dynamic collapse and dynamic response of a frame structure under seismic action. For example, Liu et al. [1] adopted time-history response analysis method and described the transition from static to vibration and then to collapse. These researchers also presented measures for improving the seismic performance of a frame structure. Liu et al. [2] analyzed three main bearing capacity reduction processes of a 10-layer frame structure, namely, elastic work, cracking, and collapse. These researchers actualized the entire process of damaging building frame structures and identified key points in structures under a severe earthquake. Liu and Chen also recommended measures for improving the seismic performance of a frame structure. Huang et al. [3] discussed the process of collecting waveform data from artificial blasting events and extracting relatively regular waveform characteristics from a natural seismic wave. The differences between blasting and natural seismic waves were identified and explored in detail. Jiang et al. [4] analyzed seismic fragility assessment of RC moment-resisting frames designed according to the current Chinese seismic design code. Yang and Gao [5] determined the out-of-plane collapse process of a filler wall under rare earthquake action through numerical simulation. These researchers confirmed that a frame filler wall falls off easily when no construction measures are undertaken; the wall then collapses under rare earthquake action. Zhang and Huang [6] studied the vibration response of a particle to rock and soil mass during an explosion by establishing a mine blasting model in the context of mining engineering. Kim and Kang [7] analyzed super tall mega frame buildings by using a multi-level condensation method. A research group (Cheng and Su [8]; Cheng and Wang [9],) also elaborated on the failure mechanism and dynamic response of a lining structure and soil mass structure surrounding a tunnel with a curved wall. Jiang et al. [10] analyzed damage-control seismic design of moment-resisting RC frame buildings. Ko et al. [11] evaluated seismic behavior of RC moment resisting frame with masonry infill walls. Research group [12] used MATLAB to examine the dynamic response of concrete frame structure under a blasting demolition environment, suggesting that moderate explosive energy should be used in demolition engineering to decrease the influence on surrounding buildings. Zhou et al. [13] conducted the test on peak acceleration amplification of soil layer in artificial blast wave. Wang et al. [14] studied the dynamic response of the frame construction building to the open-pit blasting seismic waves. The result shows that frequent blasting vibration causes the stress concentration between the wall and the beam. It is necessary to completely take into account the dynamic response difference between the frame construction building and the masonry filler wall so as to reduce the frequency of blast induced vibration to the minimum extent. Coffield and Adeli [15] studied the irregular steel building structures subjected to blast loading.

Domestic and foreign scholars have extensively studied the blasting parameters in mining areas and the influence of blasting on surrounding buildings. Lu et al. [16] examined internal force redistribution in a steel-concrete structure, as well as the relative defense mechanism caused by vehicle collision and the failure load-bearing columns during an explosion. Hao and $\mathrm{Wu}[17]$ studied the dynamic response of an RC frame structure to underground motion caused by an underground explosion, they eventually established a discrete model. By conducting an actual survey on an open gold mine in Cangshang and by analyzing slope stability, blasting vibration strength, and uneven surface settlement, Zhan et al. [18] determined that the main reason for civil house cracking is the significant difference in the compressibilities of foundation soil. An uneven surface settlement results in house cracking. Shi et al. [19] analyzed the influence of vibrations from open-pit mining blasting on neighboring buildings and compared the degree of influence of various factors using grey relation method in combination with engineering practice. Wu et al. [20] studied a blasting vibration appraisal method and calculated the safe distance based on the degree of influence of excavation blasting on adjacent buildings in the Shuibuya Quarry. Tan et al. [21] measured the vibration of a three-layer frame structure that lies on the slope of an open-pit mine boundary near the slope shoulder as a result of construction site blasting. Chen $e t$ al. [22] studied the building dynamic response under explosion load. Hao et al. [23] studied the reliability of RC column and frame with FRP strengthening subjected to explosive load. And the effectiveness of FRP strengthening of the RC column on their blast load-carrying capacities was demonstrated. These researchers established a damage accumulation model of the reinforced concrete (RC) member and in the overall structure under the action of frequent blasting vibration. The results showed that the damage is greater than the structural integrity, and that the weakest parts of the frame structure under frequent blasting vibration are located at the top floor and on top of the side column positioned at the bottom of the structure. Yan et al. [24] assessed the seismic impact on residences during blasting excavation of a large-scale rock slope in China. These researchers also discussed the response caused by ground motion at different frequencies, damage characteristics, the spatial variations in ground motion, and the soil consolidation interactions during the structural response.

Although international scholars have investigated numerous studies on blasting vibration, their studies mainly 
focused on the influencing factors that influence building damage, impact scope evaluation, and the dynamic response of the rock mass itself. In sum, most scholars have analyzed the dynamic response of an $\mathrm{RC}$ frame structure to explosions, but studies are limited to the explosive action of the building itself. Moreover, existing works do not consider the influence of explosions on the surrounding buildings. Therefore, the present study focuses on the dynamic response of a surrounding frame structure under the action of a blasting vibration wave caused by mining, and in order to compare and analyze, the frame structures that with different floor number were selected. The findings of this research could serve as a reference for the construction of buildings around the mining area.

\section{ANALYSIS MODEL AND BLASTING SEISMIC WAVE}

\subsection{Engineering Overview}

In order to study the dynamic response of surrounding frame structure under blasting vibration, residential buildings that with 7 and 16 stories were examined. These buildings belonged to RC frame structures. Moreover, these constructions share the same structure form and are similar in terms of other parameters. The building parameters were as follows: North-South total width was $18 \mathrm{~m}$, East-West total length was $30 \mathrm{~m}$, column spacing was $6 \mathrm{~m}$, the story height of the first floor was $3.9 \mathrm{~m}$, and the story heights of the remaining stories were $3.0 \mathrm{~m}$. Concrete strength was 30 $\mathrm{MPa}$, elastic modulus E was $30 \mathrm{GPa}$, and Poisson's ratio was 0.2 . The thickness of floor slab was $120 \mathrm{~mm}$, the column section was $600 \mathrm{~mm} \times 600 \mathrm{~mm}$, and the beam section was $550 \mathrm{~mm} \times 300 \mathrm{~mm}$. The dead load of the floor slab was 2.50 $\mathrm{kN} / \mathrm{m}^{2}$ (not including self-weight), the live load of the floor slab was $2.00 \mathrm{kN} / \mathrm{m}^{2}$, and the live load of the roof was 0.5 $\mathrm{kN} / \mathrm{m}^{2}$. Earthquake intensity was VIII, and the design earthquake group belonged to the second group. The site classification was class II. The standard floor layout of the building is shown in Fig. (1).

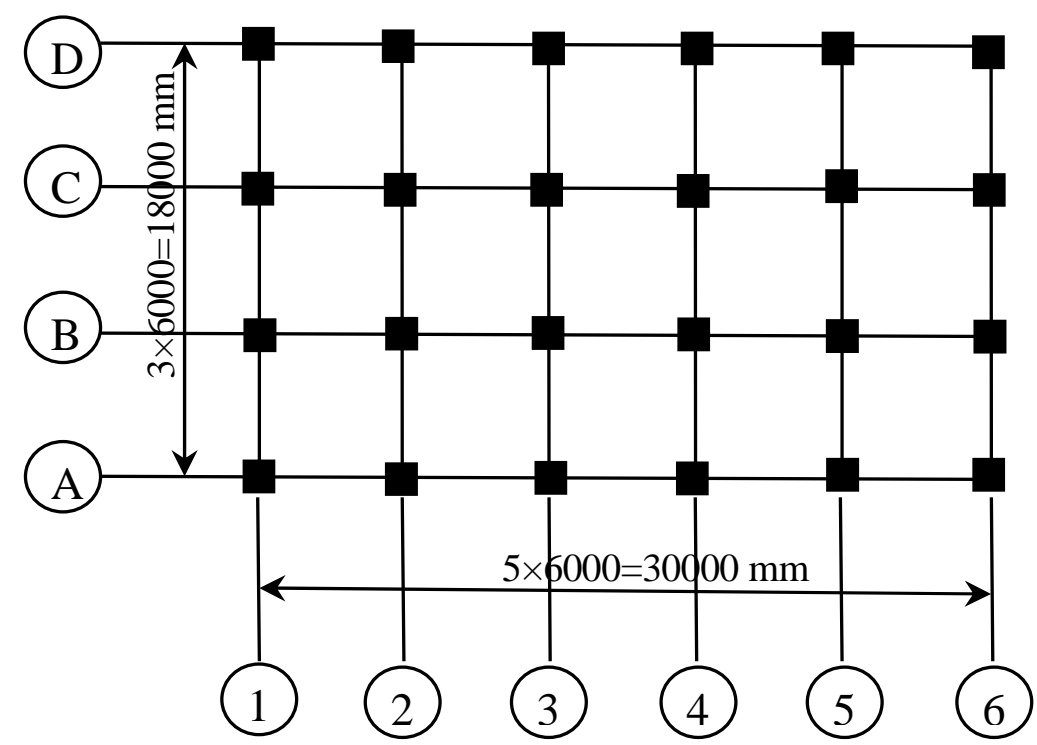

Fig. (1). Standard floor layout.

\subsection{Blasting Seismic Wave}

MATLAB software was utilized to simulate a blasting seismic wave and when considering the loading model of space explosion; the site condition indirectly through the amplitude envelope function was considered. According to the existing literature (Guo et al. [25]), the amplitude envelope function is expressed as Eq. (1), and the power spectrum function is written as Eq. (2).

$$
g(t)=m \bar{r}^{-n}\left(t / t_{0}\right)^{-a} e^{-b\left(t-t_{0}\right)} \ldots\left(a \geq 0, t \geq t_{0}, b \geq 0\right)
$$

where $\bar{r}$ is the explosion distance ratio $(\bar{r}=R / \sqrt[3]{Q}) ; Q$ is the current amount of explosive charge; $R$ is the explosive distance; $m, n, a$, and $b$ are undetermined constants; $t$ is the time; and $t_{0}$ is the delay time. 


$$
S(r, \omega)=\frac{S_{0} \omega^{4}}{r}\left[\frac{\omega e^{-k_{s} \omega^{2} r}+}{\pi r} \frac{4 S_{0} F_{0} \omega^{4}}{\left(\omega^{2}-\omega_{0}{ }^{2}\right)^{2}+4 \xi_{0}{ }^{2} \omega_{0}^{2} \omega^{2}}\right]
$$

where $S_{0}, k_{s}$, and $F_{0}$ are all constants (determined through the experiment); $r$ is the distance of the blast center; $\omega_{0}$ is the characteristic frequency $(\omega>0)$; and $\xi_{0}$ is the damping coefficient.

Fourier amplitude spectrum A $(r, \omega)$ can be calculated by power spectrum $\mathrm{S}(\mathrm{r}, \omega)$ :

$$
A(r, \omega)=[4 S(r, \omega) \Delta \omega]^{0.5}
$$

Where $\Delta \omega=2 \pi \times$ sampling frequency/FFT length, and $\Delta \omega$ is the frequency interval.

Converting Fourier amplitude spectrum $\mathrm{A}(\mathrm{r}, \omega)$ and random phase spectrum $\phi(\omega)$ to real and imaginary parts of Fourier transform, then conducting inverse Fourier transform, approximate acceleration time history a(t) of artificial seismic wave can be obtained:

$$
a(t)=F F T^{-1}\left[A(r, \omega) e^{i \phi(\omega)}\right]
$$

Where $\phi(\omega)$ is phase spectrum, it is a random number between 0 and $\pi$; $i$ is imaginary.

The final equation of artificial seismic wave can be expressed as:

$$
a_{g}(t)=a(t) g(t)
$$

According to existing literature (Lin [26]; Huang [27], $m=448.53, n=2.03, C=665.4, \omega=100, a=60, b=80$, $S=7.5 \times 10^{-8}, k_{s}=4.35 \times 10-5$, and $F=9.1 \times 10^{-6}$.

The explosion wave has a considerable relation to the explosion amount and the explosion distance. The timehistory curves of blasting seismic acceleration are constructed at the specified distances of 200, 300, and $400 \mathrm{~m}$ in the explosion amounts 200, 300, and $400 \mathrm{~kg}$, as shown in Fig. (2).

\section{MASS PARTICIPATION RATIO AND DEFORMATION DIAGRAM}

The dynamic responses of the 7-story and 16-story frame structures located at a mining field were analyzed using SAP2000 at distances of 200, 300, and $400 \mathrm{~m}$.

The period and mass participation ratio of the first three vibration modes are presented in Tables $\mathbf{1}$ and $\mathbf{2}$. UX, UY, and $\mathrm{UZ}$ are the mass participation ratios in the $\mathrm{X}, \mathrm{Y}$, and $\mathrm{Z}$ directions, respectively. Sum UX, Sum UY, and Sum UZ are the accumulation values of the mass participation ratio in the $\mathrm{X}, \mathrm{Y}$, and $\mathrm{Z}$ directions, respectively. $\mathrm{RX}, \mathrm{RY}$, and $\mathrm{RZ}$ are

\begin{tabular}{|c|c|c|c|c|c|c|c|c|c|c|c|}
\hline Distance & Order & Period & $\mathbf{U X}$ & $\mathbf{U Y}$ & $\mathbf{U Z}$ & $\begin{array}{c}\text { Sum } \\
\text { UX }\end{array}$ & $\begin{array}{c}\text { Sum } \\
\text { UY }\end{array}$ & $\begin{array}{c}\text { Sum } \\
\text { UZ }\end{array}$ & RX & RY & $\mathbf{R Z}$ \\
\hline \multirow{3}{*}{$200 \mathrm{~m}$} & 1 & 0.57 & 0.80 & 0.00 & 0.00 & 0.80 & 0.00 & 0.00 & 0.00 & 0.67 & 0.46 \\
\hline & 2 & 0.53 & 0.00 & 0.80 & 0.00 & 0.80 & 0.80 & 0.00 & 0.34 & 0.00 & 0.12 \\
\hline & 3 & 0.18 & 0.10 & 0.00 & 0.00 & 0.90 & 0.80 & 0.00 & 0.00 & 0.00 & $\overline{0.06}$ \\
\hline \multirow{3}{*}{$300 \mathrm{~m}$} & 1 & 0.57 & 0.80 & 0.00 & 0.00 & 0.80 & 0.00 & 0.00 & 0.00 & 0.67 & 0.46 \\
\hline & 2 & 0.53 & 0.00 & 0.80 & 0.00 & 0.80 & 0.80 & 0.00 & 0.34 & 0.00 & 0.12 \\
\hline & 3 & 0.18 & 0.10 & 0.00 & 0.00 & 0.90 & 0.80 & 0.00 & 0.00 & 0.00 & 0.06 \\
\hline \multirow{3}{*}{$400 \mathrm{~m}$} & 1 & 0.57 & 0.80 & 0.00 & 0.00 & 0.80 & 0.00 & 0.00 & 0.00 & 0.67 & 0.46 \\
\hline & 2 & 0.53 & 0.00 & 0.80 & 0.00 & 0.80 & 0.80 & 0.00 & 0.34 & 0.00 & 0.12 \\
\hline & 3 & 0.18 & 0.10 & 0.00 & 0.00 & 0.90 & 0.80 & 0.00 & 0.00 & 0.00 & 0.06 \\
\hline
\end{tabular}
the mass participation ratios circling the $\mathrm{X}, \mathrm{Y}$, and $\mathrm{Z}$ axes, respectively. The deformations of the structures under different modes are highlighted in Tables 3 and $\mathbf{4}$.

Table 1. Mass participation ratios of the 7-story frame structure. 


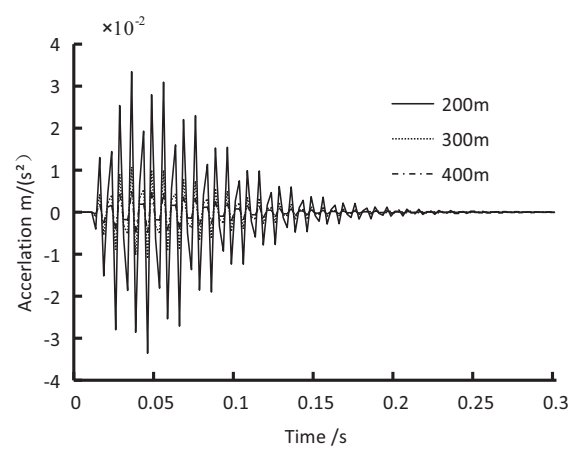

(a) The explosion amount of $200 \mathrm{~kg}$

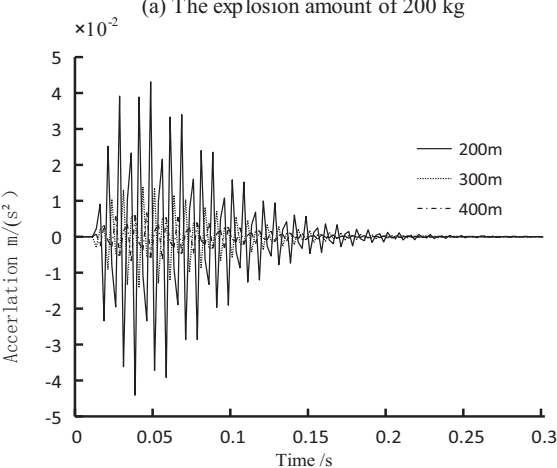

(b) The explosion amount of $300 \mathrm{~kg}$

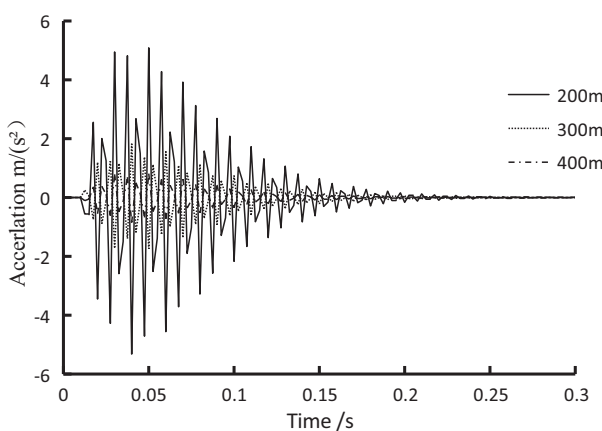

Fig. (2). Time-history curves of acceleration with different blasting vibration distances and different explosion amounts.

Table 2. Mass participation ratios of the 16-story frame structure.

\begin{tabular}{|c|c|c|c|c|c|c|c|c|c|c|c|}
\hline Distance & Order & Period & $\mathbf{U X}$ & UY & $\mathbf{U Z}$ & $\begin{array}{c}\text { Sum } \\
\text { UX }\end{array}$ & $\begin{array}{c}\text { Sum } \\
\text { UY }\end{array}$ & $\begin{array}{c}\text { Sum } \\
\text { UZ }\end{array}$ & $\mathbf{R X}$ & RY & RZ \\
\hline \multirow{3}{*}{$200 \mathrm{~m}$} & 1 & 1.37 & 0.79 & 0.00 & 0.00 & 0.79 & 0.00 & 0.00 & 0.00 & 0.90 & 0.46 \\
\hline & 2 & 1.26 & 0.00 & 0.80 & 0.00 & 0.79 & 0.80 & 0.00 & 0.71 & 0.00 & 0.12 \\
\hline & 3 & 0.45 & 0.10 & 0.00 & 0.00 & 0.89 & 0.80 & 0.00 & 0.00 & 0.00 & 0.06 \\
\hline \multirow{3}{*}{$300 \mathrm{~m}$} & 1 & 1.37 & 0.79 & 0.00 & 0.00 & 0.79 & 0.00 & 0.00 & 0.00 & 0.90 & 0.46 \\
\hline & 2 & 1.26 & 0.00 & 0.80 & 0.00 & 0.79 & 0.80 & 0.00 & 0.71 & 0.00 & 0.12 \\
\hline & 3 & 0.45 & 0.10 & 0.00 & 0.00 & 0.89 & 0.80 & 0.00 & 0.00 & 0.00 & 0.06 \\
\hline \multirow{3}{*}{$400 \mathrm{~m}$} & 1 & 1.37 & 0.79 & 0.00 & 0.00 & 0.79 & 0.00 & 0.00 & 0.00 & 0.90 & 0.46 \\
\hline & 2 & 1.26 & 0.00 & 0.80 & 0.00 & 0.79 & 0.80 & 0.00 & 0.71 & 0.00 & 0.12 \\
\hline & 3 & 0.45 & 0.10 & 0.00 & 0.00 & 0.89 & 0.80 & 0.00 & 0.00 & 0.00 & 0.06 \\
\hline
\end{tabular}


Table 3. Deformations of the 7-story frame structure.

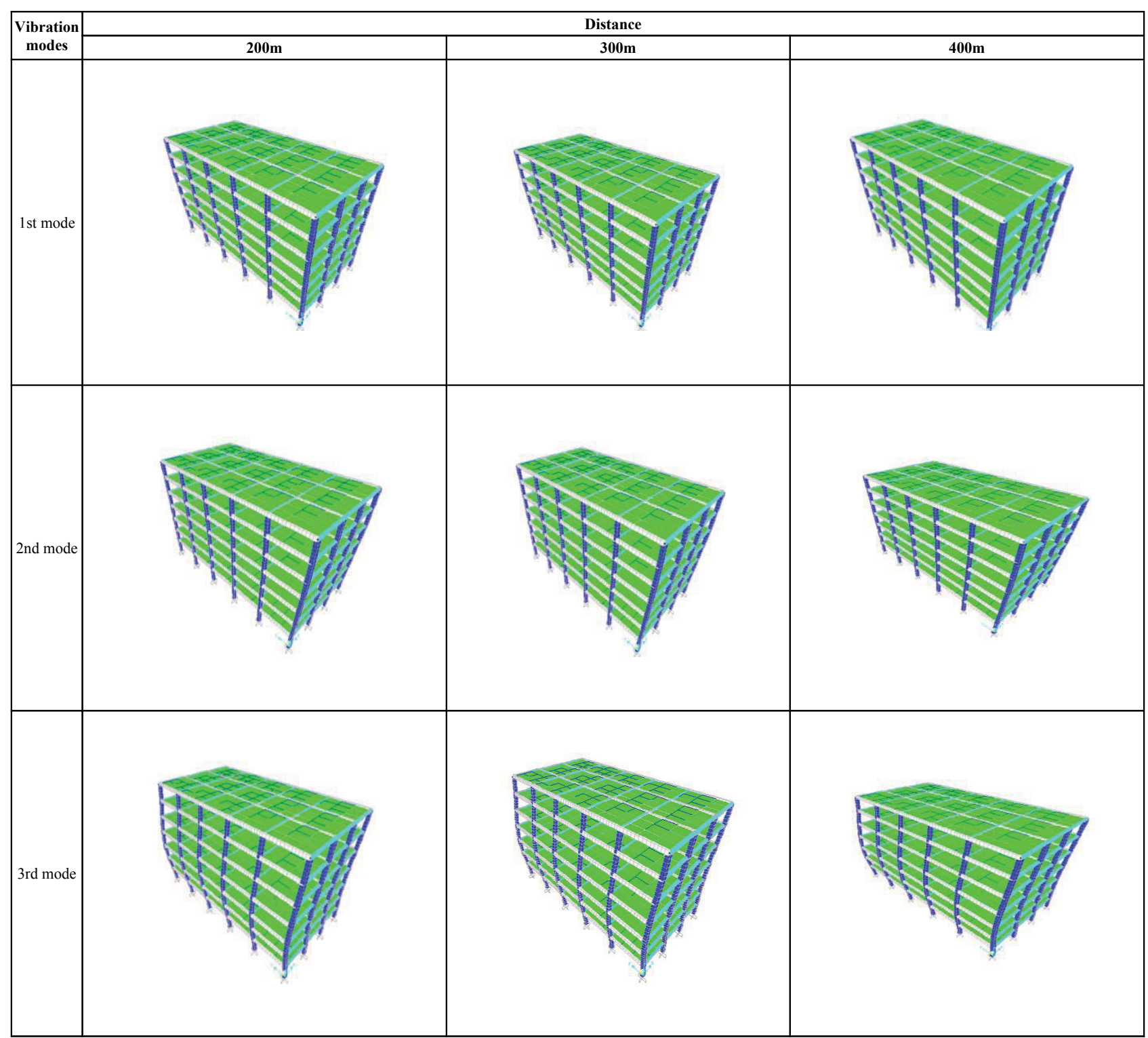

Table 4. Deformations of the 16-story frame structure.

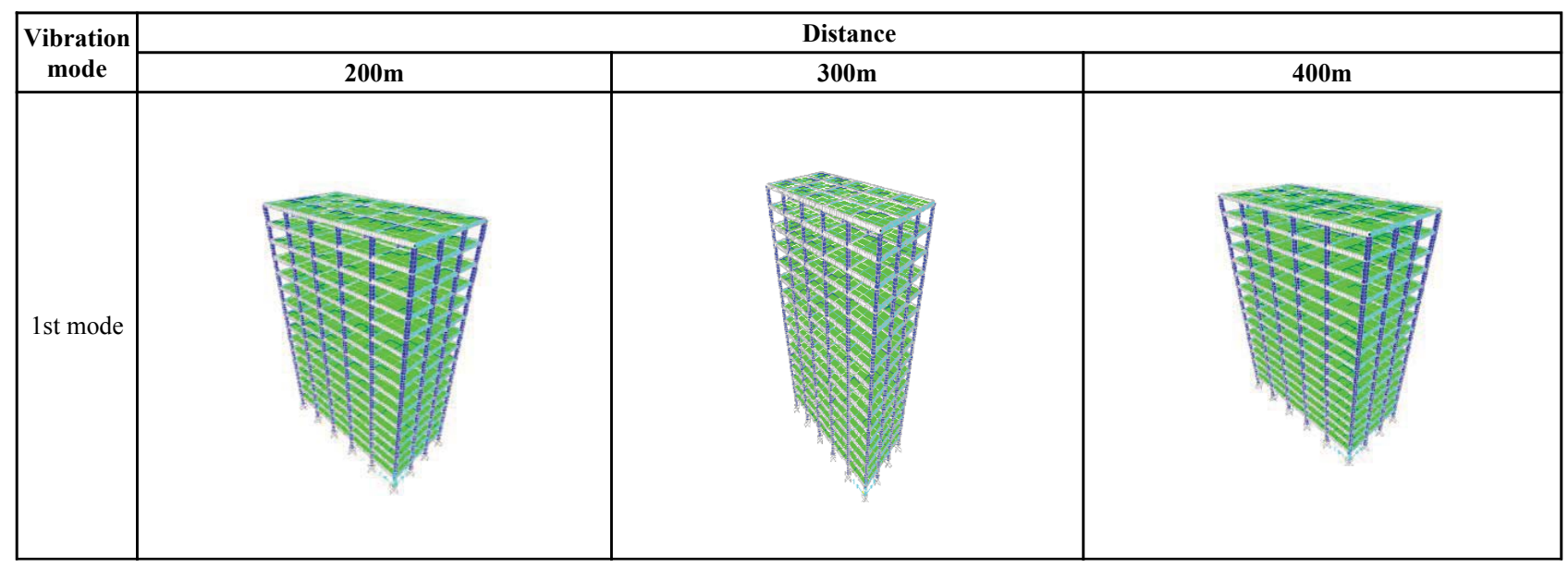




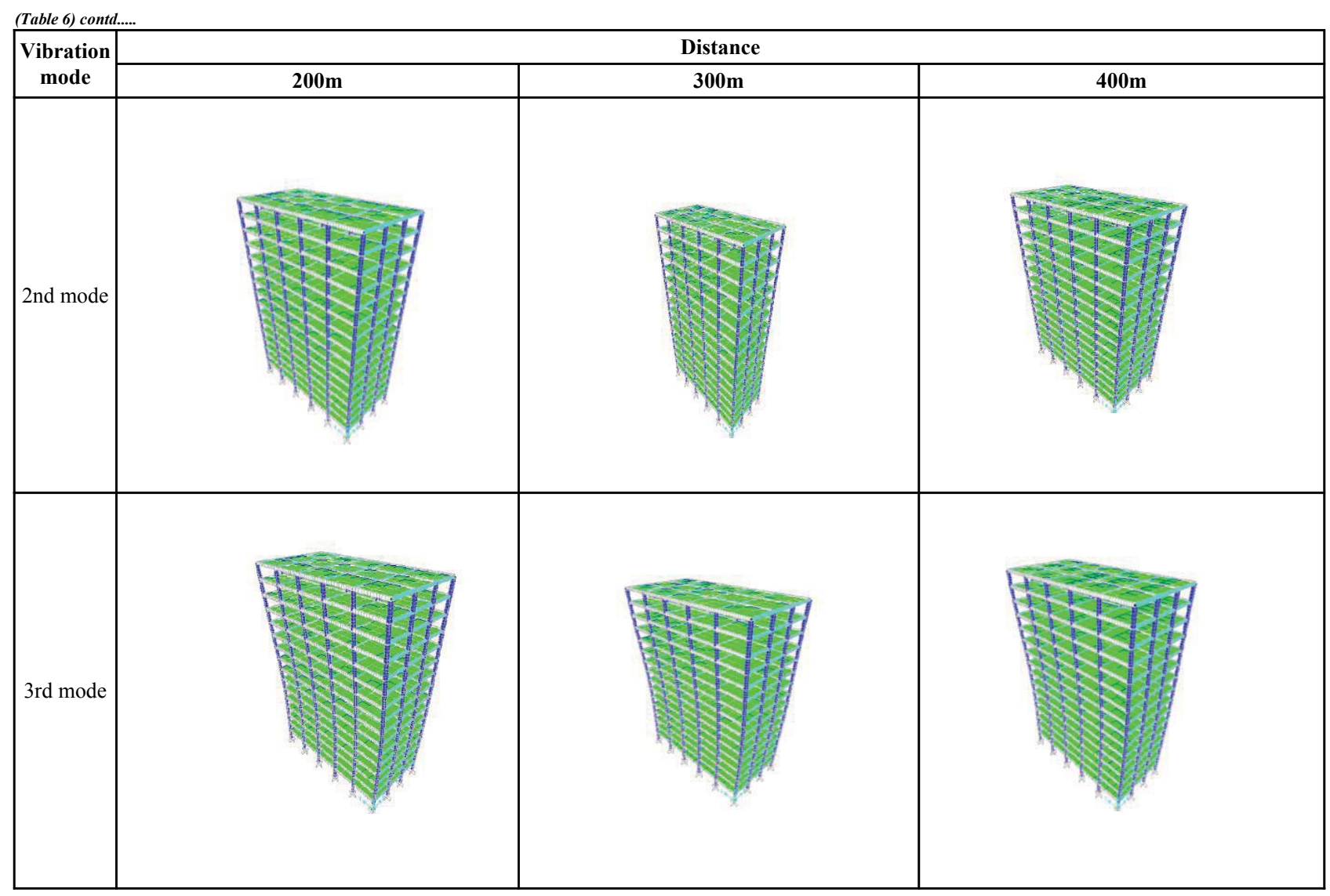

As can be seen from Table 1, the three mode periods of the 7 -story frame structure are $0.53 \mathrm{~s}, 0.57 \mathrm{~s}$ and $0.18 \mathrm{~s}$, respectively. First of all, for the first mode, UX is 0.8 , UY is $0, \mathrm{UZ}$ is $0, \mathrm{Rx}$ is $0, \mathrm{RY}$ is 0.67 and RZ is 0.46 , indicating that the structure is translational along the $\mathrm{X}$ axis and torsional around the $\mathrm{Y}$ axis and $\mathrm{Z}$ axis. Similarly, we can determine the second mode is translational along the $\mathrm{Y}$ axis and torsional around the $\mathrm{X}$ axis and $\mathrm{Z}$ axis, and the third mode is translational along the $\mathrm{Z}$ axis and torsional around the $\mathrm{X}$ axis and $\mathrm{Y}$ axis.

As can be seen from Table 2 , the three mode periods of 16-story frame structure are $1.26 \mathrm{~s}, 0.45 \mathrm{~s}$ and $1.37 \mathrm{~s}$, respectively. First of all, for the first mode, the structure is translational along the $\mathrm{X}$ axis and torsional around the $\mathrm{Y}$ axis and $\mathrm{Z}$ axis, the second mode is translational along the $\mathrm{Y}$ axis and torsional around the $\mathrm{X}$ axis and $\mathrm{Z}$ axis, and the third mode is translational along the $\mathrm{Z}$ axis and torsional around the $\mathrm{X}$ axis and $\mathrm{Y}$ axis

As can be seen from Tables $\mathbf{3}$ and $\mathbf{4}$, the deformation of 7-story frame structure is significantly greater than that of 16-story frame structure under the same blast wave. Both the 7-storey and 16-story frame structures, blasting distance is closer, the overall deformation of structure is larger. On the contrary, blasting distance is farther, the overall structure deformation is smaller.

\section{DYNAMIC RESPONSES}

\subsection{Displacement}

Tables 5 to $\mathbf{1 0}$ list the floor displacements and story drifts of the 7-story and 16-story frame structures under the action of different amount of explosion per blasting field, respectively, their distances from the explosion source are 200 $\mathrm{m}, 300 \mathrm{~m}$ and $400 \mathrm{~m}$., their amount of explosive are $200 \mathrm{~kg}, 300 \mathrm{~kg}$ and $400 \mathrm{~kg}$, respectively.

Table 5. Floor displacements of the 7-story frame structure when the amount of explosion charge is $200 \mathrm{~kg}$.

\begin{tabular}{|c|c|c|c|c|c|c|}
\hline \multirow[b]{2}{*}{ Floors } & \multicolumn{2}{|c|}{$200 \mathrm{~m}$} & \multicolumn{2}{|c|}{$300 \mathrm{~m}$} & \multicolumn{2}{|c|}{$400 \mathrm{~m}$} \\
\hline & $\begin{array}{c}\text { Floor displacement } \\
\text { (m) }\end{array}$ & $\begin{array}{l}\text { Story drift } \\
\text { (m) }\end{array}$ & $\begin{array}{c}\text { Floor displacement } \\
\text { (m) }\end{array}$ & $\begin{array}{l}\text { Story drift } \\
\text { (m) }\end{array}$ & $\begin{array}{c}\text { Floor displacement } \\
\text { (m) }\end{array}$ & $\begin{array}{l}\text { Story drift } \\
\text { (m) }\end{array}$ \\
\hline 1 & $1.021 \mathrm{E}-02$ & $1.021 \mathrm{E}-02$ & $9.188 \mathrm{E}-04$ & $9.188 \mathrm{E}-04$ & $8.534 \mathrm{E}-04$ & 8.534E-04 \\
\hline 2 & $1.863 \mathrm{E}-02$ & $8.420 \mathrm{E}-03$ & $1.670 \mathrm{E}-03$ & $7.512 \mathrm{E}-04$ & $1.557 \mathrm{E}-03$ & $7.036 \mathrm{E}-04$ \\
\hline 3 & $2.514 \mathrm{E}-02$ & $6.510 \mathrm{E}-03$ & $2.258 \mathrm{E}-03$ & $5.880 \mathrm{E}-04$ & $2.101 \mathrm{E}-03$ & $5.440 \mathrm{E}-04$ \\
\hline
\end{tabular}


(Table 5) contd.....

\begin{tabular}{|c|c|c|c|c|c|c|}
\hline \multirow{3}{*}{ Floors } & \multicolumn{2}{|c|}{$\mathbf{2 0 0} \mathbf{~ m}$} & \multicolumn{2}{c|}{$\mathbf{3 0 0} \mathbf{~ m}$} & \multicolumn{2}{c|}{$\mathbf{4 0 0} \mathbf{~ m}$} \\
\cline { 2 - 7 } & $\begin{array}{c}\text { Floor displacement } \\
(\mathbf{m})\end{array}$ & $\begin{array}{c}\text { Story drift } \\
(\mathbf{m})\end{array}$ & $\begin{array}{c}\text { Floor displacement } \\
(\mathbf{m})\end{array}$ & $\begin{array}{c}\text { Story drift } \\
(\mathbf{m})\end{array}$ & $\begin{array}{c}\text { Floor displacement } \\
(\mathbf{m})\end{array}$ & $\begin{array}{c}\text { Story drift } \\
(\mathbf{m})\end{array}$ \\
\hline 4 & $3.046 \mathrm{E}-02$ & $5.320 \mathrm{E}-03$ & $2.735 \mathrm{E}-03$ & $4.770 \mathrm{E}-04$ & $2.545 \mathrm{E}-03$ & $4.440 \mathrm{E}-04$ \\
\hline 5 & $3.850 \mathrm{E}-02$ & $8.040 \mathrm{E}-03$ & $3.458 \mathrm{E}-03$ & $7.230 \mathrm{E}-04$ & $3.217 \mathrm{E}-03$ \\
\hline 6 & $4.360 \mathrm{E}-02$ & $5.100 \mathrm{E}-03$ & $3.910 \mathrm{E}-03$ & $4.520 \mathrm{E}-04$ & $3.643 \mathrm{E}-03$ & $4.720 \mathrm{E}-04$ \\
\hline 7 & $4.559 \mathrm{E}-02$ & $1.990 \mathrm{E}-03$ & $4.088 \mathrm{E}-03$ & $1.780 \mathrm{E}-04$ & $3.809 \mathrm{E}-03$ \\
\hline
\end{tabular}

Table 6. Floor displacements of the 16-story frame structure when the amount of explosion charge is $200 \mathrm{~kg}$.

\begin{tabular}{|c|c|c|c|c|c|c|}
\hline \multirow[b]{2}{*}{ Floor } & \multicolumn{2}{|c|}{$200 \mathrm{~m}$} & \multicolumn{2}{|c|}{$300 \mathrm{~m}$} & \multicolumn{2}{|c|}{$400 \mathrm{~m}$} \\
\hline & $\begin{array}{l}\text { Floor displacement } \\
\text { (m) }\end{array}$ & $\begin{array}{l}\text { Story drift } \\
\text { (m) }\end{array}$ & $\begin{array}{l}\text { Floor displacement } \\
\text { (m) }\end{array}$ & $\begin{array}{l}\text { Story drift } \\
\text { (m) }\end{array}$ & $\begin{array}{l}\text { Floor displacement } \\
\text { (m) }\end{array}$ & $\begin{array}{l}\text { Story drift } \\
\text { (m) }\end{array}$ \\
\hline 1 & $5.641 \mathrm{E}-03$ & $5.641 \mathrm{E}-03$ & $2.490 \mathrm{E}-03$ & $2.490 \mathrm{E}-03$ & $3.858 \mathrm{E}-03$ & $3.858 \mathrm{E}-03$ \\
\hline 2 & $1.133 \mathrm{E}-02$ & $6.490 \mathrm{E}-03$ & $4.989 \mathrm{E}-03$ & $2.881 \mathrm{E}-03$ & $4.081 \mathrm{E}-03$ & $2.230 \mathrm{E}-04$ \\
\hline 3 & $1.782 \mathrm{E}-02$ & $6.530 \mathrm{E}-03$ & $7.870 \mathrm{E}-03$ & $2.890 \mathrm{E}-03$ & $4.323 \mathrm{E}-03$ & $2.420 \mathrm{E}-04$ \\
\hline 4 & $2.435 \mathrm{E}-02$ & $5.680 \mathrm{E}-03$ & $1.076 \mathrm{E}-02$ & $2.890 \mathrm{E}-03$ & $5.661 \mathrm{E}-03$ & $1.338 \mathrm{E}-03$ \\
\hline 5 & $3.003 \mathrm{E}-02$ & $4.900 \mathrm{E}-03$ & $1.328 \mathrm{E}-02$ & $2.520 \mathrm{E}-03$ & $5.703 \mathrm{E}-03$ & $4.200 \mathrm{E}-05$ \\
\hline 6 & $3.493 \mathrm{E}-02$ & $4.830 \mathrm{E}-03$ & $1.542 \mathrm{E}-02$ & $2.140 \mathrm{E}-03$ & $6.314 \mathrm{E}-03$ & $6.110 \mathrm{E}-04$ \\
\hline 7 & $3.976 \mathrm{E}-02$ & $4.990 \mathrm{E}-03$ & $1.753 \mathrm{E}-02$ & $2.110 \mathrm{E}-03$ & $6.476 \mathrm{E}-03$ & $1.620 \mathrm{E}-04$ \\
\hline 8 & $4.475 \mathrm{E}-02$ & $1.500 \mathrm{E}-04$ & $1.974 \mathrm{E}-02$ & $2.210 \mathrm{E}-03$ & $6.583 \mathrm{E}-03$ & $1.070 \mathrm{E}-04$ \\
\hline 9 & $4.490 \mathrm{E}-02$ & $3.580 \mathrm{E}-03$ & $2.134 \mathrm{E}-02$ & $1.600 \mathrm{E}-03$ & $6.906 \mathrm{E}-03$ & $3.230 \mathrm{E}-04$ \\
\hline 10 & $4.848 \mathrm{E}-02$ & $1.820 \mathrm{E}-03$ & $2.187 \mathrm{E}-02$ & $5.300 \mathrm{E}-04$ & $7.315 \mathrm{E}-03$ & $4.090 \mathrm{E}-04$ \\
\hline 11 & $5.030 \mathrm{E}-02$ & $1.930 \mathrm{E}-03$ & $2.216 \mathrm{E}-02$ & $2.900 \mathrm{E}-04$ & $7.380 \mathrm{E}-03$ & $6.500 \mathrm{E}-05$ \\
\hline 12 & $5.223 \mathrm{E}-02$ & $1.630 \mathrm{E}-03$ & $2.301 \mathrm{E}-02$ & $8.500 \mathrm{E}-04$ & 7.394E-03 & $1.400 \mathrm{E}-05$ \\
\hline 13 & $5.386 \mathrm{E}-02$ & $1.340 \mathrm{E}-03$ & $2.363 \mathrm{E}-02$ & $6.200 \mathrm{E}-04$ & $7.626 \mathrm{E}-03$ & $2.320 \mathrm{E}-04$ \\
\hline 14 & $5.520 \mathrm{E}-02$ & $1.600 \mathrm{E}-04$ & $2.374 \mathrm{E}-02$ & $1.100 \mathrm{E}-04$ & $7.792 \mathrm{E}-03$ & $1.660 \mathrm{E}-04$ \\
\hline 15 & $5.536 \mathrm{E}-02$ & $6.200 \mathrm{E}-04$ & $2.434 \mathrm{E}-02$ & $6.000 \mathrm{E}-04$ & $7.950 \mathrm{E}-03$ & $1.580 \mathrm{E}-04$ \\
\hline 16 & $5.598 \mathrm{E}-02$ & $4.848 \mathrm{E}-03$ & $2.468 \mathrm{E}-02$ & $3.400 \mathrm{E}-04$ & $8.109 \mathrm{E}-03$ & $1.590 \mathrm{E}-04$ \\
\hline
\end{tabular}

Table 7. Floor displacements of the 7-story frame structure when the amount of explosion charge is $300 \mathrm{~kg}$.

\begin{tabular}{|c|c|c|c|c|c|c|}
\hline \multirow[b]{2}{*}{ Floors } & \multicolumn{2}{|c|}{$200 \mathrm{~m}$} & \multicolumn{2}{|c|}{$300 \mathrm{~m}$} & \multicolumn{2}{|c|}{$400 \mathrm{~m}$} \\
\hline & $\begin{array}{c}\text { Floor displacement } \\
\text { (m) }\end{array}$ & $\begin{array}{l}\text { Story drift } \\
\text { (m) }\end{array}$ & $\begin{array}{c}\text { Floor displacement } \\
(\mathbf{m})\end{array}$ & $\begin{array}{l}\text { Story drift } \\
\text { (m) }\end{array}$ & $\begin{array}{l}\text { Floor displacement } \\
(\mathrm{m})\end{array}$ & $\begin{array}{l}\text { Story drift } \\
\text { (m) }\end{array}$ \\
\hline 1 & $1.142 \mathrm{E}-02$ & $1.142 \mathrm{E}-02$ & $9.711 \mathrm{E}-03$ & $9.711 \mathrm{E}-03$ & $8.852 \mathrm{E}-04$ & $8.852 \mathrm{E}-04$ \\
\hline 2 & $2.082 \mathrm{E}-02$ & $9.400 \mathrm{E}-03$ & $1.771 \mathrm{E}-02$ & $7.999 \mathrm{E}-03$ & $1.615 \mathrm{E}-03$ & $7.298 \mathrm{E}-04$ \\
\hline 3 & $2.811 \mathrm{E}-02$ & $7.290 \mathrm{E}-03$ & $2.391 \mathrm{E}-02$ & $6.200 \mathrm{E}-03$ & $2.179 \mathrm{E}-03$ & $5.640 \mathrm{E}-04$ \\
\hline 4 & $3.404 \mathrm{E}-02$ & $5.930 \mathrm{E}-03$ & $2.896 \mathrm{E}-02$ & $5.050 \mathrm{E}-03$ & $2.640 \mathrm{E}-03$ & $4.610 \mathrm{E}-04$ \\
\hline 5 & $4.303 \mathrm{E}-02$ & $8.990 \mathrm{E}-03$ & $3.661 \mathrm{E}-02$ & $7.650 \mathrm{E}-03$ & $3.337 \mathrm{E}-03$ & $6.970 \mathrm{E}-04$ \\
\hline 6 & $4.873 \mathrm{E}-02$ & $5.700 \mathrm{E}-03$ & $4.145 \mathrm{E}-02$ & $4.840 \mathrm{E}-03$ & $3.779 \mathrm{E}-03$ & $4.420 \mathrm{E}-04$ \\
\hline 7 & $5.095 \mathrm{E}-02$ & $2.220 \mathrm{E}-03$ & $4.334 \mathrm{E}-02$ & $1.890 \mathrm{E}-03$ & $3.951 \mathrm{E}-03$ & $1.720 \mathrm{E}-04$ \\
\hline
\end{tabular}

Table 8. Floor displacements of the 16-story frame structure when the amount of explosion charge is $300 \mathrm{~kg}$.

\begin{tabular}{|c|c|c|c|c|c|c|}
\hline \multirow[b]{2}{*}{ Floor } & \multicolumn{2}{|c|}{$200 \mathrm{~m}$} & \multicolumn{2}{|c|}{$300 \mathrm{~m}$} & \multicolumn{2}{|c|}{$400 \mathrm{~m}$} \\
\hline & $\begin{array}{l}\text { Floor displacement } \\
\text { (m) }\end{array}$ & $\begin{array}{l}\text { Story drift } \\
\text { (m) }\end{array}$ & $\begin{array}{c}\text { Floor displacement } \\
(\mathbf{m})\end{array}$ & $\begin{array}{l}\text { Story drift } \\
\text { (m) }\end{array}$ & $\begin{array}{l}\text { Floor displacement } \\
(\mathrm{m})\end{array}$ & $\begin{array}{l}\text { Story drift } \\
\text { (m) }\end{array}$ \\
\hline 1 & $1.332 \mathrm{E}-02$ & $1.332 \mathrm{E}-02$ & $6.356 \mathrm{E}-04$ & $6.356 \mathrm{E}-04$ & 7.697E-04 & 7.697E-04 \\
\hline 2 & $2.660 \mathrm{E}-02$ & $1.328 \mathrm{E}-02$ & $1.908 \mathrm{E}-03$ & $1.273 \mathrm{E}-03$ & $1.530 \mathrm{E}-03$ & $7.630 \mathrm{E}-04$ \\
\hline 3 & $4.211 \mathrm{E}-02$ & $1.551 \mathrm{E}-02$ & $3.918 \mathrm{E}-03$ & $2.010 \mathrm{E}-03$ & $2.165 \mathrm{E}-03$ & $6.350 \mathrm{E}-04$ \\
\hline 4 & $5.756 \mathrm{E}-02$ & $1.545 \mathrm{E}-02$ & $6.665 \mathrm{E}-03$ & $2.747 \mathrm{E}-03$ & $2.604 \mathrm{E}-03$ & $4.390 \mathrm{E}-04$ \\
\hline 5 & $7.106 \mathrm{E}-02$ & $1.350 \mathrm{E}-02$ & $1.006 \mathrm{E}-02$ & $3.391 \mathrm{E}-03$ & $3.161 \mathrm{E}-03$ & $5.570 \mathrm{E}-04$ \\
\hline 6 & $8.253 \mathrm{E}-02$ & $1.147 \mathrm{E}-02$ & $1.399 \mathrm{E}-02$ & $3.939 \mathrm{E}-03$ & $3.827 \mathrm{E}-03$ & $6.660 \mathrm{E}-04$ \\
\hline 7 & $9.367 \mathrm{E}-02$ & $1.114 \mathrm{E}-02$ & $1.847 \mathrm{E}-02$ & $4.476 \mathrm{E}-03$ & $4.331 \mathrm{E}-03$ & $5.040 \mathrm{E}-04$ \\
\hline 8 & $1.055 \mathrm{E}-01$ & $1.183 \mathrm{E}-02$ & $2.351 \mathrm{E}-02$ & $5.039 \mathrm{E}-03$ & $4.988 \mathrm{E}-03$ & $6.570 \mathrm{E}-04$ \\
\hline
\end{tabular}


(Table 8) contd.....

\begin{tabular}{|c|c|c|c|c|c|c|}
\hline \multirow[b]{2}{*}{ Floor } & \multicolumn{2}{|c|}{$200 \mathrm{~m}$} & \multicolumn{2}{|c|}{$300 \mathrm{~m}$} & \multicolumn{2}{|c|}{$400 \mathrm{~m}$} \\
\hline & $\begin{array}{c}\text { Floor displacement } \\
\text { (m) }\end{array}$ & $\begin{array}{l}\text { Story drift } \\
\text { (m) }\end{array}$ & $\begin{array}{c}\text { Floor displacement } \\
\text { (m) }\end{array}$ & $\begin{array}{l}\text { Story drift } \\
\text { (m) }\end{array}$ & $\begin{array}{c}\text { Floor displacement } \\
(\mathbf{m})\end{array}$ & $\begin{array}{l}\text { Story drift } \\
\text { (m) }\end{array}$ \\
\hline 9 & $1.137 \mathrm{E}-01$ & $8.200 \mathrm{E}-03$ & $2.909 \mathrm{E}-02$ & $5.584 \mathrm{E}-03$ & $5.551 \mathrm{E}-03$ & $5.630 \mathrm{E}-04$ \\
\hline 10 & $1.169 \mathrm{E}-01$ & $3.200 \mathrm{E}-03$ & $3.515 \mathrm{E}-02$ & $6.056 \mathrm{E}-03$ & $6.202 \mathrm{E}-03$ & $6.510 \mathrm{E}-04$ \\
\hline 11 & $1.183 \mathrm{E}-01$ & $1.400 \mathrm{E}-03$ & $4.145 \mathrm{E}-02$ & $6.298 \mathrm{E}-03$ & $6.802 \mathrm{E}-03$ & $6.000 \mathrm{E}-04$ \\
\hline 12 & $1.228 \mathrm{E}-01$ & $4.500 \mathrm{E}-03$ & $4.766 \mathrm{E}-02$ & $6.213 \mathrm{E}-03$ & $7.403 \mathrm{E}-03$ & $6.010 \mathrm{E}-04$ \\
\hline 13 & $1.262 \mathrm{E}-01$ & $3.400 \mathrm{E}-03$ & $5.369 \mathrm{E}-02$ & $6.031 \mathrm{E}-03$ & $7.993 \mathrm{E}-03$ & $5.900 \mathrm{E}-04$ \\
\hline 14 & $1.268 \mathrm{E}-01$ & $6.000 \mathrm{E}-04$ & $5.957 \mathrm{E}-02$ & $5.873 \mathrm{E}-03$ & $8.537 \mathrm{E}-03$ & 5.440E-04 \\
\hline 15 & $1.300 \mathrm{E}-01$ & $3.200 \mathrm{E}-03$ & $6.522 \mathrm{E}-02$ & $5.656 \mathrm{E}-03$ & $8.981 \mathrm{E}-03$ & $4.440 \mathrm{E}-04$ \\
\hline 16 & $1.318 \mathrm{E}-01$ & $1.800 \mathrm{E}-03$ & $7.066 \mathrm{E}-02$ & $5.443 \mathrm{E}-03$ & $9.286 \mathrm{E}-03$ & $3.050 \mathrm{E}-04$ \\
\hline
\end{tabular}

Table 9. Floor displacements of the 7-story frame structure when the amount of explosion charge is $400 \mathrm{~kg}$.

\begin{tabular}{|c|c|c|c|c|c|c|}
\hline \multirow[b]{2}{*}{ Floors } & \multicolumn{2}{|c|}{$200 \mathrm{~m}$} & \multicolumn{2}{|c|}{$300 \mathrm{~m}$} & \multicolumn{2}{|c|}{$400 \mathrm{~m}$} \\
\hline & $\begin{array}{c}\text { Floor displacement } \\
(\mathrm{m})\end{array}$ & $\begin{array}{l}\text { Story drift } \\
\text { (m) }\end{array}$ & $\begin{array}{c}\text { Floor displacement } \\
(\mathrm{m})\end{array}$ & $\begin{array}{l}\text { Story drift } \\
\text { (m) }\end{array}$ & $\begin{array}{c}\text { Floor displacement } \\
(\mathrm{m})\end{array}$ & $\begin{array}{l}\text { Story drift } \\
\text { (m) }\end{array}$ \\
\hline 1 & $1.747 \mathrm{E}-02$ & $1.747 \mathrm{E}-02$ & $1.032 \mathrm{E}-02$ & $1.032 \mathrm{E}-02$ & $9.318 \mathrm{E}-03$ & $9.318 \mathrm{E}-03$ \\
\hline 2 & $3.345 \mathrm{E}-02$ & $1.598 \mathrm{E}-02$ & $1.883 \mathrm{E}-02$ & $8.510 \mathrm{E}-03$ & $1.700 \mathrm{E}-02$ & $7.682 \mathrm{E}-03$ \\
\hline 3 & $5.077 \mathrm{E}-02$ & $1.732 \mathrm{E}-02$ & $2.541 \mathrm{E}-02$ & $6.580 \mathrm{E}-03$ & $2.294 \mathrm{E}-02$ & $5.940 \mathrm{E}-03$ \\
\hline 4 & $6.259 \mathrm{E}-02$ & $1.182 \mathrm{E}-02$ & $3.078 \mathrm{E}-02$ & $5.370 \mathrm{E}-03$ & $2.779 \mathrm{E}-02$ & $4.850 \mathrm{E}-03$ \\
\hline 5 & $7.113 \mathrm{E}-02$ & $8.540 \mathrm{E}-03$ & $3.891 \mathrm{E}-02$ & $8.130 \mathrm{E}-03$ & $3.513 \mathrm{E}-02$ & $7.340 \mathrm{E}-03$ \\
\hline 6 & $8.330 \mathrm{E}-02$ & $1.217 \mathrm{E}-02$ & $4.406 \mathrm{E}-02$ & $5.150 \mathrm{E}-03$ & $3.978 \mathrm{E}-02$ & $4.650 \mathrm{E}-03$ \\
\hline 7 & $9.224 \mathrm{E}-02$ & $8.940 \mathrm{E}-03$ & $4.607 \mathrm{E}-02$ & $2.010 \mathrm{E}-03$ & $4.159 \mathrm{E}-02$ & $1.810 \mathrm{E}-03$ \\
\hline
\end{tabular}

Table 10. Floor displacements of the 16-story frame structure when the amount of explosion charge is $400 \mathrm{~kg}$.

\begin{tabular}{|c|c|c|c|c|c|c|}
\hline \multirow{2}{*}{ Floor } & \multicolumn{2}{|c|}{$\mathbf{2 0 0} \mathbf{~ m}$} & \multicolumn{2}{c|}{$\mathbf{3 0 0} \mathbf{m}$} & \multicolumn{2}{c|}{$\mathbf{4 0 0} \mathbf{~ m}$} \\
\cline { 2 - 7 } & $\begin{array}{c}\text { Floor displacement } \\
(\mathbf{m})\end{array}$ & $\begin{array}{c}\text { Story drift } \\
(\mathbf{m})\end{array}$ & $\begin{array}{c}\text { Floor displacement } \\
(\mathbf{m})\end{array}$ & $\begin{array}{c}\text { Story drift } \\
(\mathbf{m})\end{array}$ & $\begin{array}{c}\text { Floor displacement } \\
(\mathbf{m})\end{array}$ & $\begin{array}{c}\text { Story drift } \\
(\mathbf{m})\end{array}$ \\
\hline 1 & $2.792 \mathrm{E}-02$ & $2.792 \mathrm{E}-02$ & $7.540 \mathrm{E}-03$ & $7.540 \mathrm{E}-03$ & $4.472 \mathrm{E}-03$ & $4.472 \mathrm{E}-03$ \\
\hline 2 & $5.542 \mathrm{E}-02$ & $2.750 \mathrm{E}-02$ & $1.497 \mathrm{E}-02$ & $7.430 \mathrm{E}-03$ & $8.880 \mathrm{E}-03$ & $4.408 \mathrm{E}-03$ \\
\hline 3 & $7.809 \mathrm{E}-02$ & $2.267 \mathrm{E}-02$ & $2.110 \mathrm{E}-02$ & $6.130 \mathrm{E}-03$ & $1.251 \mathrm{E}-02$ & $3.630 \mathrm{E}-03$ \\
\hline 4 & $9.368 \mathrm{E}-02$ & $1.559 \mathrm{E}-02$ & $2.530 \mathrm{E}-02$ & $4.200 \mathrm{E}-03$ & $1.501 \mathrm{E}-02$ & $2.500 \mathrm{E}-03$ \\
\hline 5 & $1.132 \mathrm{E}-01$ & $1.952 \mathrm{E}-02$ & $3.056 \mathrm{E}-02$ & $5.260 \mathrm{E}-03$ & $1.814 \mathrm{E}-02$ & $3.130 \mathrm{E}-03$ \\
\hline 6 & $1.370 \mathrm{E}-01$ & $2.380 \mathrm{E}-02$ & $3.700 \mathrm{E}-02$ & $6.440 \mathrm{E}-03$ & $2.194 \mathrm{E}-02$ & $3.800 \mathrm{E}-03$ \\
\hline 7 & $1.547 \mathrm{E}-01$ & $1.770 \mathrm{E}-02$ & $4.174 \mathrm{E}-02$ & $4.740 \mathrm{E}-03$ & $2.478 \mathrm{E}-02$ & $2.840 \mathrm{E}-03$ \\
\hline 8 & $1.781 \mathrm{E}-01$ & $2.340 \mathrm{E}-02$ & $4.804 \mathrm{E}-02$ & $6.300 \mathrm{E}-03$ & $2.852 \mathrm{E}-02$ & $3.740 \mathrm{E}-03$ \\
\hline 9 & $1.978 \mathrm{E}-01$ & $1.970 \mathrm{E}-02$ & $5.337 \mathrm{E}-02$ & $5.330 \mathrm{E}-03$ & $3.169 \mathrm{E}-02$ & $3.170 \mathrm{E}-03$ \\
\hline 10 & $2.210 \mathrm{E}-01$ & $2.320 \mathrm{E}-02$ & $5.963 \mathrm{E}-02$ & $6.260 \mathrm{E}-03$ & $3.541 \mathrm{E}-02$ & $3.720 \mathrm{E}-03$ \\
\hline 11 & $2.424 \mathrm{E}-01$ & $2.140 \mathrm{E}-02$ & $6.541 \mathrm{E}-02$ & $5.780 \mathrm{E}-03$ & $3.884 \mathrm{E}-02$ & $3.430 \mathrm{E}-03$ \\
\hline 12 & $2.639 \mathrm{E}-01$ & $2.150 \mathrm{E}-02$ & $7.120 \mathrm{E}-02$ & $5.790 \mathrm{E}-03$ & $4.227 \mathrm{E}-02$ & $3.430 \mathrm{E}-03$ \\
\hline 13 & $2.851 \mathrm{E}-01$ & $2.120 \mathrm{E}-02$ & $7.692 \mathrm{E}-02$ & $5.720 \mathrm{E}-03$ & $4.567 \mathrm{E}-02$ & $3.400 \mathrm{E}-03$ \\
\hline 14 & $3.048 \mathrm{E}-01$ & $1.970 \mathrm{E}-02$ & $8.223 \mathrm{E}-02$ & $5.310 \mathrm{E}-03$ & $4.882 \mathrm{E}-02$ & $3.150 \mathrm{E}-03$ \\
\hline 15 & $3.211 \mathrm{E}-01$ & $1.630 \mathrm{E}-02$ & $8.662 \mathrm{E}-02$ & $4.390 \mathrm{E}-03$ & $5.143 \mathrm{E}-02$ & $2.610 \mathrm{E}-03$ \\
\hline 16 & $3.324 \mathrm{E}-01$ & $1.130 \mathrm{E}-02$ & $8.667 \mathrm{E}-02$ & $5.000 \mathrm{E}-05$ & $5.323 \mathrm{E}-02$ & $1.800 \mathrm{E}-03$ \\
\hline
\end{tabular}

From Tables 5 to 10, the maximum floor displacements of 7-story and 16-story frame structures can be obtained and the results are shown in Fig. (3).

According to Tables $\mathbf{5}$ and $\mathbf{6}$, the vibration response of the top story is maximum under the action of blasting wave. Fig. (3) shows that with the increase of the explosion distance, the blasting vibration response decreases.

From the Tables 5 and 6, the biggest story drift of 7-story frame structure appear on the 2nd story, 7th story and 1st story under the action of $200 \mathrm{~m}, 300 \mathrm{~m}$ and $400 \mathrm{~m}$ explosion distance; and the biggest story drift of 16-story frame structure all appear on the 10th story under the action of $200 \mathrm{~m}, 300 \mathrm{~m}$ and $400 \mathrm{~m}$ explosion distances.

The maximum story drifts of 7-story and 16-story frame structures are shown in (Fig. 4). 


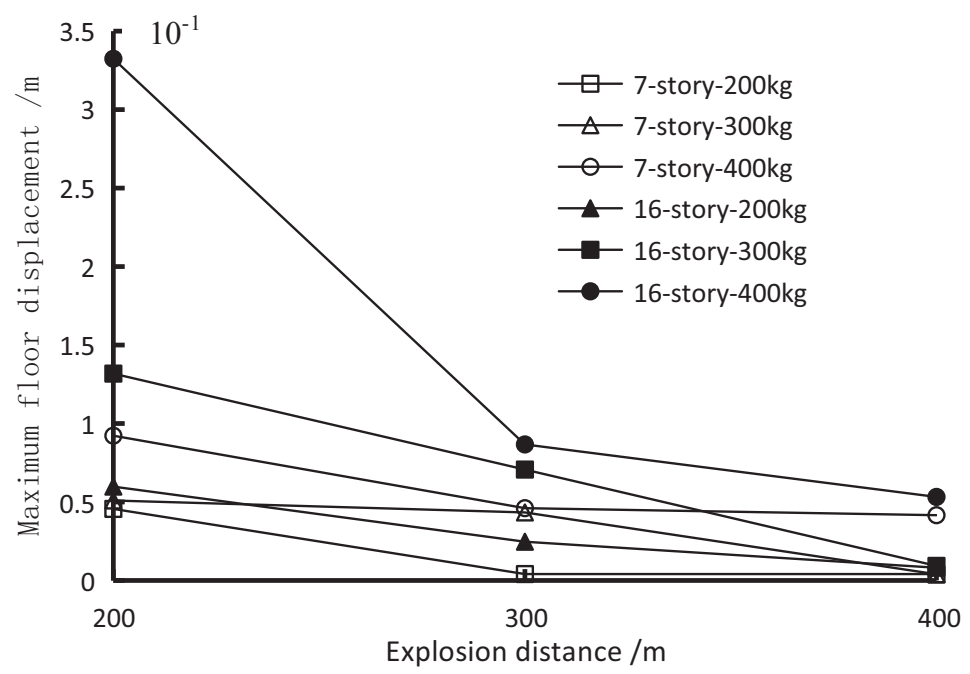

Fig. (3). Maximum floor displacement of frame structures.

From Fig. (4), the story drifts of 7-story and 16-story frame structures are the maximum under the action of $200 \mathrm{~m}$ explosion distance. With the increase of explosion distance, the story drifts gradually reduce. At the distance of $200 \mathrm{~m}$ when the amount of explosion charge is 300 and $400 \mathrm{~kg}$, the story drifts of 16-story are all bigger than that of the 7story. While at the distance of 300 and $400 \mathrm{~m}$ when the amount of explosion charge is 300 and $400 \mathrm{~kg}$, the story drifts of 7-story are all bigger than that of the 16-story. And when the amount of explosion charge is $200 \mathrm{~kg}$, the corresponding results of the two structures are on the contrary at different distances. It indicates that the amount of explosion has a greater impact on the story drifts when the explosion distance is small and the height of the structer becomes the main factor as the explosion distance increases. When the amount of explosion charge is $200 \mathrm{~kg}$, the explosion distance has bigger influence on the lower structure. According to the existing codes in China, the inter-story displacement angle of frame structure is less than $1 / 550$ by analyzing the results, the 7 -story and 16-story frame structures can't be damaged under the action of $200 \mathrm{~m}, 300 \mathrm{~m}$ and $400 \mathrm{~m}$ explosion distances.

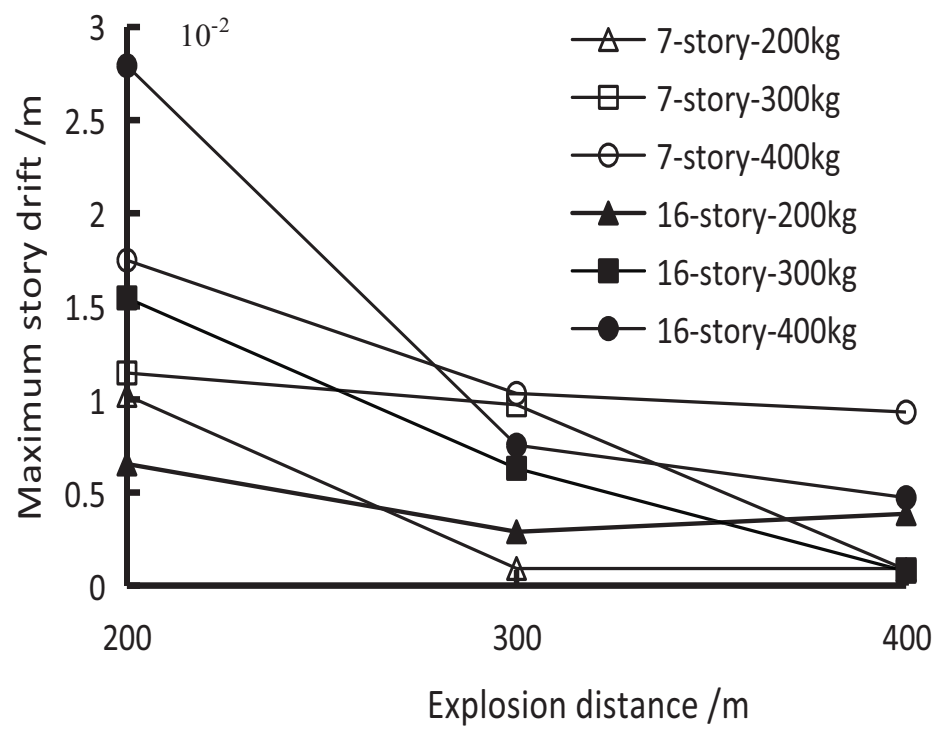

Fig. (4). Maximum story drift of frame structure. 


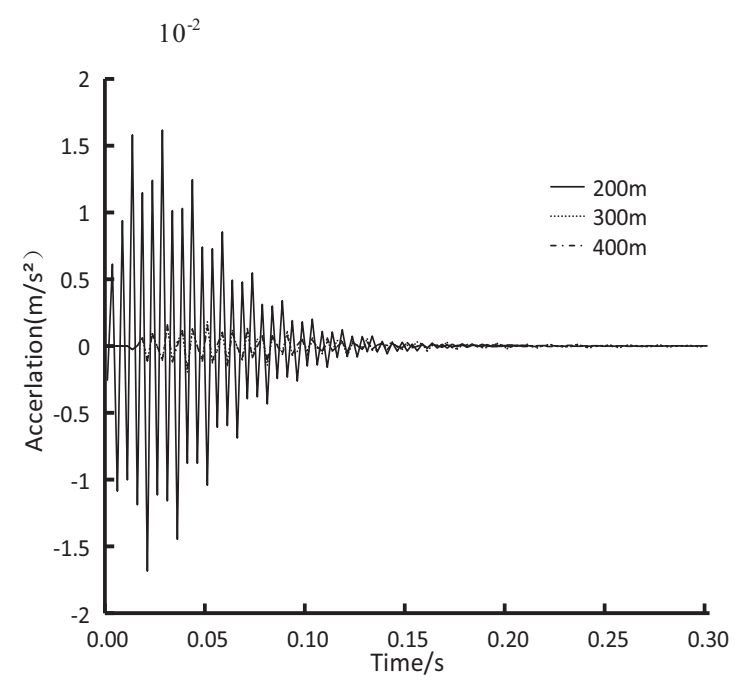

Fig. (5). show the time-history curves of acceleration on the top story at different blasting distances.

As can be seen from Figs. (3 and 4), under the same explosion source distance, the lower the structure height, the greater the maximum displacement of the floor, the story drift of the lower structure caused by the explosion is often greater than the higher structure. The maximum floor displacement and maximum story drift caused by blasting vibration are all weakened with increase of explosion source distance.

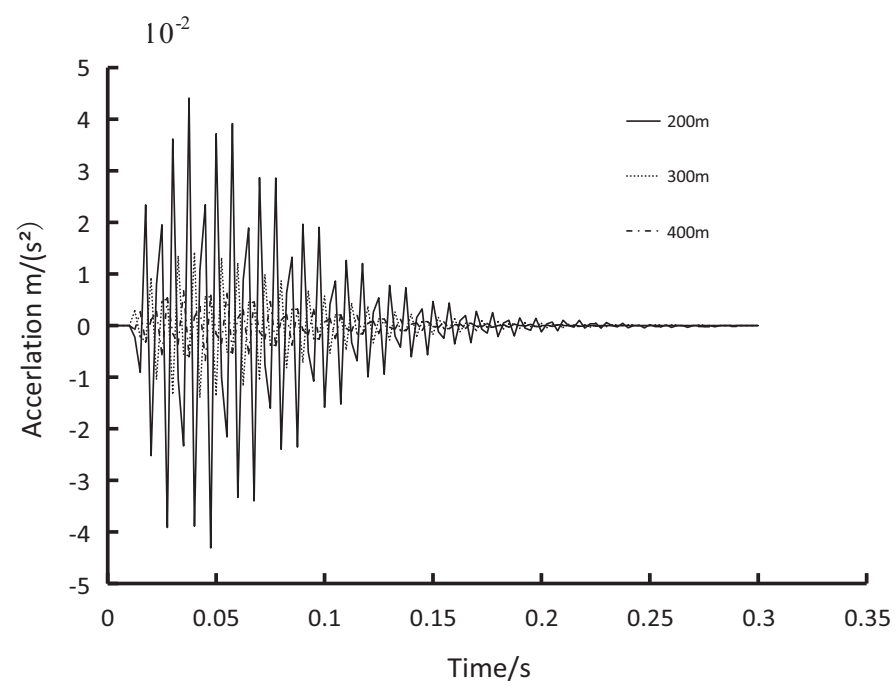

Fig. (6). Acceleration time-history curve of the 7 th story when the amount of explosivon charge is $300 \mathrm{~kg}$.

\subsection{Seismic Deformation Checking}

Under the action of frequent earthquake, the floor displacement should satisfy the following formula:

$$
\Delta \mathrm{u}_{\mathrm{e}} \leq\left[\theta_{e}\right] h
$$

Where $\Delta u_{e}$ is the maximum floor displacement of the structure produced by the standard value of the frequent seismic action, in the frame structure, $\left[\theta_{\mathrm{e}}\right]=1 / 550, h$ is the height of the frame structure. 
Table 11 list the floor displacement of the 7-story and 16-story frame structures under the action of different amount of explosion and the value of $\Delta \mathrm{u}_{\mathrm{e}}$.

Table 11. Floor displacements of the two frame structures and the value of $\Delta u_{e^{*}}$

\begin{tabular}{|c|c|c|c|c|c|}
\hline \multirow{2}{*}{$\begin{array}{c}\text { Explosion amount } \\
(\mathrm{kg})\end{array}$} & \multirow{2}{*}{\multicolumn{2}{|c|}{$\Delta u_{e}(\mathrm{~m})$}} & $200 \mathrm{~m}$ & $300 \mathrm{~m}$ & $400 \mathrm{~m}$ \\
\hline & & & \begin{tabular}{|c|} 
Floor displacement \\
$(\mathrm{m})$
\end{tabular} & $\begin{array}{c}\text { Floor displacement } \\
(\mathrm{m})\end{array}$ & $\begin{array}{c}\text { Floor displacement } \\
(\mathrm{m})\end{array}$ \\
\hline \multirow{2}{*}{200} & 7 -story & $3.980 \mathrm{E}-02$ & $4.559 \mathrm{E}-02$ & $4.088 \mathrm{E}-03$ & 3.809E-03 \\
\hline & 16-story & $8.890 \mathrm{E}-02$ & $5.980 \mathrm{E}-02$ & $2.468 \mathrm{E}-02$ & $8.109 \mathrm{E}-03$ \\
\hline \multirow{2}{*}{300} & 7-story & $3.980 \mathrm{E}-02$ & $5.095 \mathrm{E}-02$ & 4.334E-02 & $3.951 \mathrm{E}-03$ \\
\hline & 16-story & $8.890 \mathrm{E}-02$ & $1.318 \mathrm{E}-01$ & $7.066 \mathrm{E}-02$ & $9.286 \mathrm{E}-03$ \\
\hline \multirow{2}{*}{400} & 7-story & $3.980 \mathrm{E}-02$ & $9.224 \mathrm{E}-02$ & 4.607E-02 & $4.159 \mathrm{E}-02$ \\
\hline & \begin{tabular}{|l}
16 -story \\
\end{tabular} & $8.890 \mathrm{E}-02$ & $3.324 \mathrm{E}-01$ & $8.667 \mathrm{E}-02$ & $5.323 \mathrm{E}-02$ \\
\hline
\end{tabular}

From Table 11, we can find that the 7-story frame structure is damaged while the 16-story frame structure not at the $200 \mathrm{~m}$ and $300 \mathrm{~m}$ explosive distance of $200 \mathrm{~kg}$ explosive amount. And we can find the same phenomenon at the $300 \mathrm{~m}$ explosive distance under the action of $300 \mathrm{~kg}$ explosive amount and $300 \mathrm{~m}, 400 \mathrm{~m}$ explosive distance respectively under the action of explosive amount of $400 \mathrm{~kg}$. It means that the safe distance of the 7 -story frame structure is larger than that of 16-story.

Figs. (5 to 10) show the time-history curves of acceleration on the top story at different blasting distances.

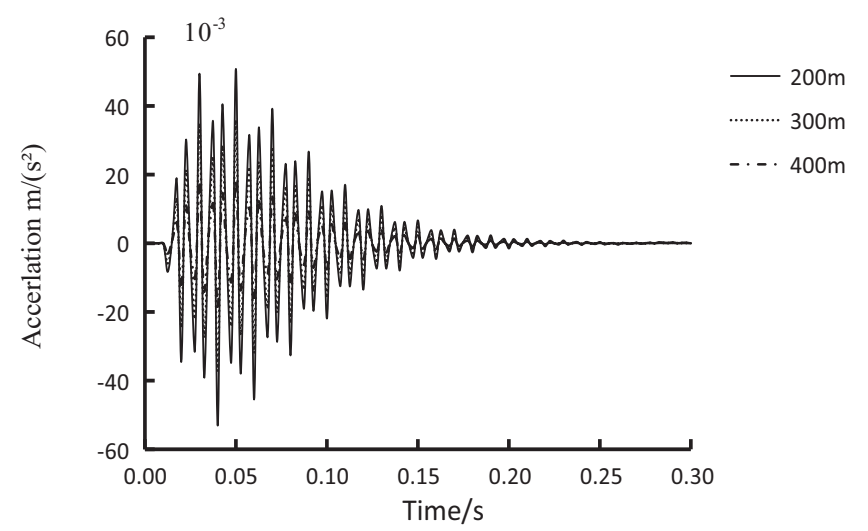

Fig. (7). Acceleration time-history curve of the 7th story when the amount of explosion charge is $400 \mathrm{~kg}$.

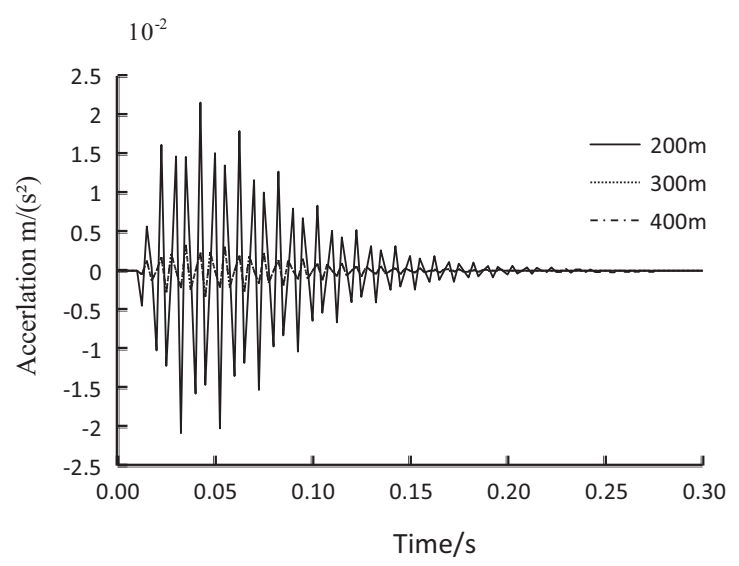

Fig. (8). Acceleration time-history curve of the 16th story when the amount of explosion charge is $200 \mathrm{~kg}$. 


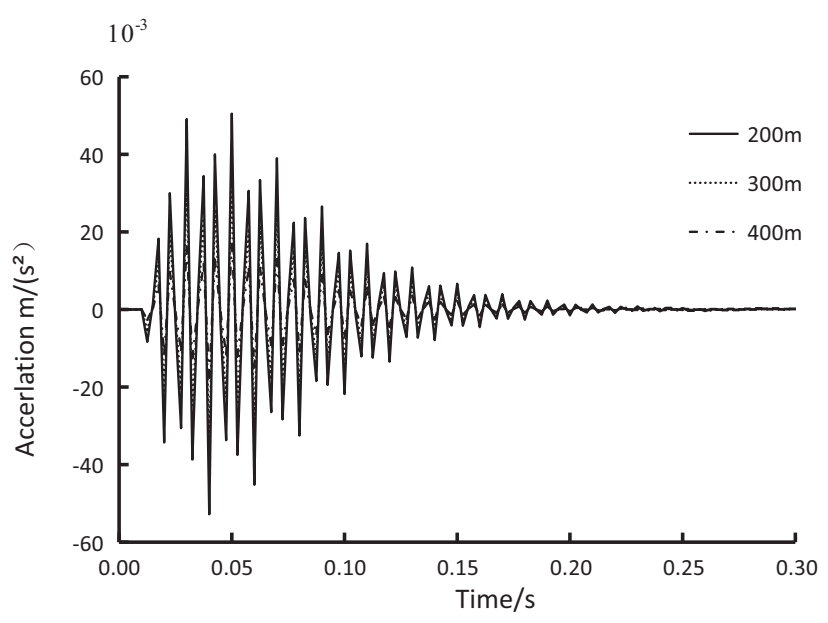

Fig. (9). Acceleration time-history curve of the 16th story when the amount of explosion charge is $300 \mathrm{~kg}$.

Figs. (5 to 10) show that the acceleration of top story of the frame structure caused by explosion source at $200 \mathrm{~m}$ is greater than at $300 \mathrm{~m}$ and $400 \mathrm{~m}$. The maximum accelerations at the top stories of the two structures appear at approximately $0.05 \mathrm{~s}$ under the action of different blasting seismic waves. Moreover, the acceleration amplitudes of the 7-story and 16-story are almost equal under the same amount of explosion and the same explosion distance. This result indicates that the height of building has minimal influence on the acceleration response caused by explosion.

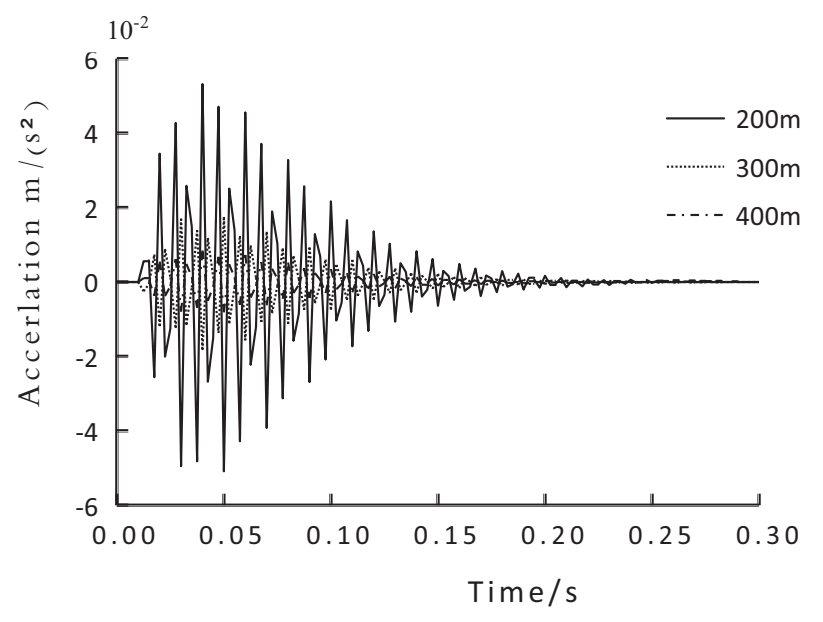

Fig. (10). Acceleration time-history curve of the 16th story when the amount of explosion charge is $400 \mathrm{~kg}$.

\section{CONCLUSION}

In this paper, the variables used are the amount of explosion, the explosion source distance, the height of structure. By controlling two of the variables unchanged, the corresponding datas of the other variable are compared and analyzed. And by controlling the single variable, the corresponding datas of the other two variables respectively are compared and analyzed to summarize the relationships between the three variables on influncing the dynamic response of mining blasting vibration to surrounding reinforced concrete (RC) frame structures.

1. The smaller the explosion source distance, the lower the structure, the larger the story drift, that is, in general, with the same explosive charge and small explosive source, the deformation of lower structure is larger than the higher.

2. For the 7-story and 16-story frame structures, the maximum story displacement and maximum story drift of the structure are increased with the increase of the amount of explosion, and in different amount of explosion they all show that the maximum story displacement and maximum story drift of the structure are decreased with the increase of the distance from the explosion source, when the explosion source distance is large, the dynamic 
response of the structure is very small, even can be ignored. Therefore, a safe distance for buildings should be determined according to the situation in the mining area.

3. The higher the structure is, the greater the influence of explosion source distance on the change rate of floor displacement and story drift is. The floor displacements of the two structures all have an obvious decreased trend between the explosion distances $200 \mathrm{~m}$ and $300 \mathrm{~m}$. It indicates that the change rate of floor displacement is smaller when the explosion source is farther.

4. Under the same explosion amount and the same explosion distance, the top acceleration amplitudes of the 16story frame structure is larger than the those of 7-story. This result indicates that the number of stories in buildings has little influence on acceleration response caused by blasting vibration.

\section{CONSENT FOR PUBLICATION}

Not applicable.

\section{CONFLICT OF INTEREST}

The authors declare no conflict of interest, financial or otherwise.

\section{ACKNOWLEDGEMENTS}

This paper is a part of the national natural science foundation of China (Grant number: 51368039, 51478212), and a part of the education ministry doctoral tutor foundation of China (Grant number: 20136201110003), and a part of the plan project of science and technology in Lanzhou city (Grant number: 2014-4-94).

\section{REFERENCES}

[1] H.Q. Liu, Z.G. Ni, and Y. Zhang, "Simulation analysis for the collapse response of frame structures under microseism", Science Technology and Engineering, vol. 9, pp. 472-475, 2009. March

[2] H. Liu, Z. Chen, and J. Yuan, "3-D simulation on collapse of high-rise structures under strong earthquake", Journal of Liaoning Technology University. Natural Science., vol. 29, pp. 1074-1077, 2010.

[3] H.M. Huang, Y.J. Bian, S.J. Lu, Z.F. Jiang, and R. Li, "A wavelet feature research on seismic waveforms of earthquakes and explosions", Acta Seismological Sinica, vol. 32, pp. 270-276, 2010. May

[4] H.J. Jiang, X.L. Lu, and L.Z. Chen, "Seismic fragility assessment of RC moment-resisting frames designed according to the current Chinese seismic design code", Journal of Asian Architecture and Building Engineering, vol. 11, pp. 153-160, 2012. [http://dx.doi.org/10.3130/jaabe.11.153]

[5] W. Yang, and F. Gao, "Collapse simulation and failure analysis of infilled walls for earthquake-resistant structures", Structural Engineer, vol. 28, pp. 98-101, 2012. August

[6] J.H. Zhang, and G. Huang, "Study of blasting vibration effect on HML open-pit mine slope", Blasting, vol. 29, pp. 114-118, 2012. August

[7] H.S. Kim, and J.W. Kang, "An efficient structural analysis of super tall mega frame buildings using a multi-level condensation method", Journal of Asian Architecture and Building Engineering, vol. 11, pp. 343-350, 2012. November [http://dx.doi.org/10.3130/jaabe.11.343]

[8] X.S. Cheng, and J.X. Su, "Dynamic response of soil tunnel lining structure under explosion", Chinese Journal of Applied Mechanics., vol. 29, pp. 104-108, 2012. February

[9] X.S. Cheng, and J.H. Wang, "Explosion dynamic response of soil surrounding tunnel with curve wall", Journal of Chongqing University., vol. 36, pp. 142-149, 2013. April

[10] H.J. Jiang, B. Fu, and L.Z. Chen, "Damage-control seismic design of moment-resisting RC frame buildings", Journal of Asian Architecture and Building Engineering, vol. 12, pp. 49-56, 2013. May [http://dx.doi.org/10.3130/jaabe.12.49]

[11] H. Ko, H.S. Kim, and J.W. Kang, "Evaluation of seismic behavior of RC moment resisting frame with masonry infill walls", Journal of Asian Architecture and Building Engineering, vol. 13, pp. 641-648, 2010. October [http://dx.doi.org/10.3130/jaabe.13.641]

[12] X. S. Cheng, W. Jing, and J. X. Ma, "Dynamic response of concrete frame structure under a blasting demolition environment", Electronic Journal of Geotechnical Engineering, vol. 19 (Bund. Z7), pp. 17823-17837, 2014. January

[13] X.M. Zhou, J.B. Lu, L. Luo, and T. Chen, "Test on peak acceleration amplification of soil layer in artificial blast wave", International Conference on Civil Engineering and Rock Engineering, 2015pp. 529-533

[14] Z.W. Wang, S.H. Dai, H. Gao, and Y.Z. Li, "Dynamic response of the frame construction building to the open-pit blasting seismic waves", Journal of Safety and Environment., vol. 15, pp. 111-115, 2015. 
[15] A. Coffield, and H. Adeli, "Irregular steel building structures subjected to blast loading", Journal of Civil Engineering and Management, vol. 22, pp. 17-25, 2016. January [http://dx.doi.org/10.3846/13923730.2015.1073172]

[16] H.S. Lu, E.C. Lu, and S. Altin, "Resistance mechanisms in RC building frames subjected to column failure", Journal of Structural Engineering, vol. 120, pp. 765-782, 1994. March [http://dx.doi.org/10.1061/(ASCE)0733-9445(1994)120:3(765)]

[17] H. Hao, and C.Q. Wu, "Numerical study of characteristics of underground blast induced surface ground motion and their effect on aboveground structures. Part II. effects on structural responses", Soil Dynamics and Earthquake Engineering, vol. 25, pp. 39-53, 2005. january [http://dx.doi.org/10.1016/j.soildyn.2004.08.002]

[18] Y.B. Zhan, C.A. You, and J.H. Wang, "Cracking causes analysis of rural building in open pit mine area", China Mining Magazine, vol. 15, pp. 44-46, 2006. October

[19] X.Z. Shi, H.Y. Wang, J.J. Tian, and S.R. Chen, "Sensitivity analysis of factors causing damage of residential houses by blasting vibration based on grey association analysis", Blasting, vol. 25, pp. 17-21, 2008. September

[20] X.X. Wu, X.B. Ren, and W.H. Wang, "The identifying method of the blasting vibration destruction scope on the resident houses about the quarry", Blasting, vol. 26, pp. 93-95, 2009. December

[21] W.H. Tan, M. Yu, and P.F. Zhang, "Influence of frequent blasting vibration on the performance of a frame structure", Journal of Wuhan University of Technology., vol. 32, pp. 252-256, 2010. May

[22] S.L. Chen, Q.J. Xu, Y. Long, and N. Jing, "Numerical analysis of building dynamic response under explosion load", PLA University of Science and Technology, vol. 3, pp. 74-78, 2015. November

[23] H. Hao, Z.X. Li, and Y.C. Shi, "Reliability analysis of RC columns and frame with FRP strengthening subjected to explosive load", Journal of Performance of Constructed Facilities, [Online]. Available: http://www.ascelibrary.org, vol. 30, 2016no. 2, pp. 74-78. Accessed Sept. 20 [http://dx.doi.org/10.1061/(ASCE)CF.1943-5509.0000748]

[24] P. Yan, Y.J. Zou, J.R. Zhou, W. Lu, and Y. Zhang, "Assessment of seismic impact on residences during blasting excavation of a large-scale rock slope in China", Environmental Earth Science, vol. 75, pp. 1-15, 2016. [http://dx.doi.org/10.1007/s12665-016-5747-6]

[25] S. B. Guo, Y. F. Pan, P. Z. Gao, M. Y. Wang, and Q. H. Qian, "Numerical simulation of explosion seismic waves", Explosion and Shock waves, vol. 25, pp. 335-340. July

[26] D.C. Lin, "Stochastic evolution theory and application research of explosion ground vibration", PH. D. thesis, University of Beijing Institute of Technology, Beijing, ON, China, 2001.

[27] Y.L. Huang, "Theoretical study on seismic source characteristics of blasting seismic waves", PH. D. thesis, China University of Mining and Technology, Beijing, ON, China, 2000.

\section{(C) 2017 Cheng et al.}

This is an open access article distributed under the terms of the Creative Commons Attribution 4.0 International Public License (CC-BY 4.0), a copy of which is available at: https://creativecommons.org/licenses/by/4.0/legalcode. This license permits unrestricted use, distribution, and reproduction in any medium, provided the original author and source are credited. 